\title{
Synthesis and characterization of manganese containing mesoporous bioactive glass nanoparticles for biomedical applications
}

\author{
Qaisar Nawaz ${ }^{1}$ Muhammad Atiq Ur Rehman ${ }^{1} \cdot$ Andreas Burkovski $^{2} \cdot$ Jochen Schmidt $^{3}$ - Ana M. Beltrán ${ }^{4}$. \\ Ameen Shahid ${ }^{5} \cdot$ Nina K. Alber ${ }^{2} \cdot$ Wolfgang Peukert $^{3} \cdot$ Aldo R. Boccaccini $^{1}{ }^{1}$
}

Received: 30 December 2017 / Accepted: 18 April 2018 / Published online: 8 May 2018

(c) Springer Science+Business Media, LLC, part of Springer Nature 2018

\begin{abstract}
Mesoporous bioactive glass (BG) nanoparticles based in the system: $\mathrm{SiO}_{2}-\mathrm{P}_{2} \mathrm{O}_{5}-\mathrm{CaO}-\mathrm{MnO}$ were synthesized via a modified Stöber process at various concentrations of $\mathrm{Mn}(0-7 \mathrm{~mol} \%)$. The synthesized manganese-doped BG nanoparticles were characterized in terms of morphology, composition, in vitro bioactivity and antibacterial activity. Scanning electron microscopy (SEM), transmission electron microscopy (TEM) and Brunauer-Emmett-Teller (BET) analysis confirmed that the particles had spherical morphology (mean particle size: $110 \mathrm{~nm}$ ) with disordered mesoporous structure. Energy dispersive X-ray spectroscopy (EDX) confirmed the presence of $\mathrm{Mn}, \mathrm{Ca}, \mathrm{Si}$ and $\mathrm{P}$ in the synthesized Mn-doped BG particles. Moreover, X-ray diffraction (XRD) analysis showed that Mn has been incorporated in the amorphous silica network (bioactive glass). Moreover, it was found that manganese-doped BG particles form apatite crystals upon immersion in simulated body fluid (SBF). Inductively coupled plasma atomic emission spectroscopy (ICP-OES) measurements confirmed that $\mathrm{Mn}$ is released in a sustained manner, which provided antibacterial effect against Bacillus subtilis, Pseudomonas aeruginosa and Staphylococcus aureus. The results indicate that the incorporation of $\mathrm{Mn}$ in the bioactive glass network is an effective strategy to develop novel multifunctional BG nanoparticles for bone tissue engineering.
\end{abstract}

\section{Introduction}

Bone tissue engineering (BTE) is an interdisciplinary approach, which provides strategies for the repair and regeneration of bone [1]. BTE usually involves the application of a 3D scaffold fabricated from bioactive materials,

$\triangle$ Aldo R. Boccaccini

aldo.boccaccini@ww.uni-erlangen.de

1 Department of Materials Science and Engineering, Institute of Biomaterials, University of Erlangen-Nuremberg, Cauerstr. 6, Erlangen 91058, Germany

2 Microbiology Division, University of Erlangen-Nuremberg, Staudtstr. 5, Erlangen 91058, Germany

3 Institute of Particle Technology, University of ErlangenNuremberg, Cauerstr. 4, Erlangen 91058, Germany

4 Department of Materials Science and Engineering, University of Seville, Seville 41011, Spain

5 Institute of Chemical Reaction Engineering, University of Erlangen-Nuremberg, Egerlandstr. 3, Erlangen 91058, Germany which have suitable chemical composition and mechanical properties [1-3]. Synthetic materials such as bioactive glasses, hydroxyapatite or their composites with biopolymers are used for developing BTE scaffolds, bone filling materials and coatings in biomedical implants $[4,5]$. 45S5 bioactive glass (BG) $\left(45 \mathrm{SiO}_{2}-24.5 \mathrm{Na}_{2} \mathrm{O}-24.5 \mathrm{CaO}-6\right.$ $\mathrm{P}_{2} \mathrm{O}_{5}$ (wt\%)), first developed by Hench et al. [6] more than 40 years ago, is capable to turn on the body's own regenerative system leading to new tissue formation $[7,8]$. It has been shown that the rapid mineralization induced by $45 \mathrm{~S} 5$ $\mathrm{BG}$ is due to the release of $\mathrm{Ca}$ and phosphate ions in critical concentrations, inducing bone cells to proliferate and grow [7]. Moreover, the release of $\mathrm{Si}$ species has been shown to regulate gene expression in osteoblastic cells $[9,10]$ while the angiogenic effect of 45S5 BG has been also reported $[11,12]$.

Several other types of bioactive glasses such as $58 \mathrm{~S}$ (60 $\left.\mathrm{SiO}_{2}-36 \mathrm{CaO}-4 \mathrm{P}_{2} \mathrm{O}_{5}(\mathrm{~mol} \%)\right), 1393\left(53 \mathrm{SiO}_{2}-20 \mathrm{CaO}-6\right.$ $\left.\mathrm{Na}_{2} \mathrm{O}-4 \mathrm{P}_{2} \mathrm{O}_{5}-12 \mathrm{~K}_{2} \mathrm{O}-5 \mathrm{MgO}(\mathrm{wt} \%)\right)$ and $70 \mathrm{~S} 30 \mathrm{C}(70$ $\mathrm{SiO}_{2}-30 \mathrm{CaO}(\mathrm{mol} \%)$ ) among many others have also been developed, which exhibit bioactivity and biocompatibility $[7,13,14]$. Furthermore, modifications have been done in 
the nominal 45S5 BG composition by incorporating different biologically active elements [15] such as cerium [2], gallium [16], cobalt [17], copper [18], strontium [19], lithium [20], niobium [21], boron [22] and zinc [23]. These metallic ions can enhance bioactivity and impart new biological functionalities such as angiogenic and antibacterial effects.

There is limited work related to incorporation of $\mathrm{Mn}$ in BGs. The few available studies have shown that the presence of $\mathrm{Mn}$ in the $\mathrm{BG}\left(50 \mathrm{SiO}_{2}-10 \mathrm{P}_{2} \mathrm{O}_{5}-40-\mathrm{X} \mathrm{CaO}-\mathrm{X}\right.$ $\mathrm{MnO}(\mathrm{mol} \%)$ ) network improves bone mineralization, extracellular matrix remodeling and promotes cell adhesion [24-26]. Mn influences bone remodeling because its lower content in the body relates to the rise of concentrations of calcium and phosphate. For instance, Mncontaining hydroxyapatite has been shown to improve adhesion and proliferation of osteoblastic-like cells (MC3T3E1) [27]. Furthermore, Miola et al. [28] modified melt-derived bioactive glasses in the system $\mathrm{SiO}_{2}-\mathrm{P}_{2} \mathrm{O}_{5^{-}}$ $\mathrm{CaO}-\mathrm{MgO}-\mathrm{Na}_{2} \mathrm{O}-\mathrm{K}_{2} \mathrm{O}$ with $\mathrm{Mn}$, which exhibited potential for osteoblast growth and osteogenic activity. Sol-gel derived Mn-doped bioactive glasses were recently reported by Barrioni et al. [29]. It was observed that Mn enhanced cell proliferation and viability of osteoblastic cells, while maintaining acceptable bioactivity. Manganese exhibits antibacterial activity at low concentration against a broad spectrum of Gram-positive and Gramnegative bacteria without being toxic for cells. The antibacterial effect is primarily associated with the release of therapeutic ions in critical concentrations. Mn ions can bind to the thiol groups, which cause the death of bacteria, i.e., $\mathrm{Mn}$ ions can bind to the proteins altering their structure causing rupture of bacterial walls and eventually preventing the function of DNA associated with bacteria division and replication [30-32] (Mn has been incorporated in $\mathrm{ZnO}$ nanoparticles and different complexes for antibacterial studies [30-32]). Due to the lack of extensive studies on Mn-containing BGs it is of importance to further investigate the effect of $\mathrm{Mn}$ on in vitro bioactivity and antibacterial effect of BGs before considering the material for in vivo studies.

Two processing methods are mainly used to synthesize BGs: the traditional melt quenching process and the sol-gel technique [7]. Sol-gel derived glasses offer versatility in composition, sizes and morphologies, and the sol-gel technique is particularly suitable for synthesis of nanoparticles [7]. Furthermore, sol-gel derived BGs have improved bioactivity due to the high surface area and the presence of silanol groups, which can act as nucleation sites for the formation of apatite crystals [33, 34].

The sol-gel process is the method of choice to synthesize mesoporous bioactive glasses (MBGs), which combine the textural parameters of an ordered mesoporous matrix with high specific surface area and large pore volume. These features are especially useful in bone tissue engineering and drug delivery applications [35-37]. MBGs are promising vehicles for delivering therapeutic ions; mainly since their degradation rate and ion release capability can be adjusted through the modification of composition. The unique textural properties of these bioactive glasses enhance the bioactivity and sustain the release of biologically active molecules/ions for longer periods of time [17, 34, 38]. Different therapeutic ions including $\mathrm{Ag}^{+}, \mathrm{Sr}^{+2}, \mathrm{Cu}^{+2}$ and $\mathrm{Co}^{+2}$ have been incorporated into MBGNs, and their release has enhanced several biological functions, as reported in literature [19, 39-42].

The focus of this research work was to synthesize for the first time Mn-containing mesoporous bioactive glass nanoparticles (Mn-MBGNs) via a modified Stöber method [43, 44]. Mesoporous bioactive glass nanoparticles were initially doped with various concentrations of $\mathrm{Mn}(0,3,5,7$ (mol $\%)$ ). Preliminary in vitro bioactivity studies indicated that 5 Mn-MBNGs formed hydroxyapatite crystals upon immersion in simulated body fluid (SBF) confirming their suitability for bone tissue engineering applications. Moreover the controlled release of $\mathrm{Mn}$ ions induced an antibacterial effect without affecting the inherent bioactivity associated with MBGNs [29]. The antibacterial effect is primarily associated with the release of metallic ions in critical concentrations. The method utilized in this study determines the antibacterial effect in physiological conditions against relevant bacterial strains and pathogens. It was shown that the produced powder has the ability to confer antibacterial activity, it can induce antibacterial effect in scaffolds and coatings [29, 40, 45]. Thus the present results may pave the way for utilizing $\mathrm{Mn}$ containing mesoporous bioactive glass nanoparticles for scaffold fabrication (bone tissue engineering) and for bioactive and antibacterial coatings on metallic implants.

\section{Materials and methods}

\subsection{Materials}

Tetraethyl orthosilicate [TEOS]-99\% (Sigma), triethyl phosphate [TEP]-99\% (Aldrich, Germany), calcium nitrate [Ca $\left.\left(\mathrm{NO}_{3}\right)_{2} .4 \mathrm{H}_{2} \mathrm{O}\right]-98 \%$ (Aldrich, Germany), and manganese chloride $\left[\mathrm{MnCl}_{2} \cdot 4 \mathrm{H}_{2} \mathrm{O}\right]$-(Merck, Germany), were used as silicon, phosphorous, calcium and manganese sources, respectively. Furthermore, ethyl acetate, cetyltrimethylammonium bromide [CTAB]-(Merck, Germany), ammonium hydroxide 28\% (VWR, France), distilled water (MilliQ) and absolute ethanol- 99.8\% (Alfa Aesar, Germany) were used. All chemicals used were of analytical grade. 


\subsection{Synthesis of Mn-containing MBGNs}

Mn-MBGNs were produced using a modified Stöber process [43, 44]. Firstly, $0.56 \mathrm{~g}$ CTAB (soft template) was dissolved in $26 \mathrm{ml}$ of water under continuous stirring for $15 \mathrm{~min}$. Secondly, $8 \mathrm{ml}$ ethyl acetate was poured drop wise into the above solution. Thirdly, ammonium hydroxide (28\%) was added to maintain $\mathrm{pH}$ at 10.5 and $3 \mathrm{ml}$ of TEOS was added into the above solution under continuous stirring. Finally, calcium nitrate, TEP and manganese chloride were added step-wise followed by magnetic stirring for $30 \mathrm{~min}$. The solution was allowed to react for $3 \mathrm{~h}$. After that, the suspension was centrifuged at $7830 \mathrm{rpm}$ (Centrifuge 5430R, Eppendorf, Germany) for $10 \mathrm{~min}$ to separate particles from the parent's solvent. This step was repeated three times. Afterwards, the precipitates were dried in an oven at $60^{\circ} \mathrm{C}$ for $12 \mathrm{~h}$, followed by calcination at $700^{\circ} \mathrm{C}$ (heating rate of $2{ }^{\circ} \mathrm{C} / \mathrm{min}$ ) for $5 \mathrm{~h}$.

Moreover, it is important to mention that during the washing steps, the unreacted precursors and remaining salts are washed out which can affect the final composition. There is always a variation in the intended (initially calculated) composition and the actual (finally obtained) composition. Further studies such as X-ray fluorescence (XRF) will be helpful to determine the actual composition of the synthesized bioactive glass. However, the desired tentative compositions are given in Table 1.

\subsection{Characterization of Mn-MBGNs}

The surface morphology of the as synthesized MBGNs was analyzed by field emission scanning electron microscopy (FE-SEM, LEO 435VP, Carl Zeiss ${ }^{\mathrm{TM}}$ AG) at an energy of $5 \mathrm{kV}$ and by transmission electron microscopy (TEM, FEI Talos F200S microscope) operating at an accelerating voltage of $200 \mathrm{kV}$. For the TEM observation, samples were deposited on a Holey Carbon Film on a copper grid. SEM samples were sputtered coated (Q150/ S, Quorum Technologies $^{\mathrm{TM}}$ ) with gold prior to FESEM analysis to prevent the effect of charging. Compositional analysis of the powders was done qualitatively using energy-dispersive $\mathrm{x}$-ray spectroscopy (EDX) at $15 \mathrm{kV}$ (LEO 435VP, Carl Zeiss ${ }^{\mathrm{TM}}$

Table 1 Nominal composition of as-synthesized mesoporous bioactive glass nanoparticles

\begin{tabular}{lllll}
\hline Bioactive glass types & \multicolumn{5}{l}{ Compositions (mol \%) } \\
\cline { 2 - 5 } & $\mathrm{SiO}_{2}$ & $\mathrm{CaO}$ & $\mathrm{P}_{2} \mathrm{O}_{5}$ & $\mathrm{MnO}$ \\
\hline MBGNs & 50 & 40 & 10 & 0 \\
3 Mn-MBGNs & 50 & 37 & 10 & 3 \\
5 Mn-MBGNs & 50 & 35 & 10 & 5 \\
7 Mn-MBGNs & 50 & 33 & 10 & 7 \\
\hline
\end{tabular}

AG). Fourier Transform Infrared spectroscopy (Nicolet 6700 FTIR spectrometer, Thermo Scientific ${ }^{\mathrm{TM}}$, USA) was carried out in transmission mode at the wavenumber ranging from 4000 to $400 \mathrm{~cm}^{-1}$ at a resolution of $4 \mathrm{~cm}^{-1}$. Xray diffraction (XRD) analysis was performed using X-ray diffractometer (D8 Advance, Brucker ${ }^{\mathrm{TM}}$ ) in the range of $10^{\circ}$ to $60^{\circ}$ with a step size of $0.010^{\circ}$ and dwell time of $1^{\circ}$ per minute. $\mathrm{Cu} \mathrm{K} \alpha$ radiation was used. Prior to XRD measurements, the powders were dispersed on low background silicon wafer (Bruker AXS). $\mathrm{N}_{2}$-physisorption was performed in a Quadrasorb ${ }^{\mathrm{TM}}$ SI gas adsorption analyzer at $77 \mathrm{~K}$ for surface area and pore size analyses. Prior to the measurements, the pre-treatment of samples was carried out for $12 \mathrm{~h}$ at $300^{\circ} \mathrm{C}$ in vacuum.

\subsection{In vitro bioactivity assessment}

The bioactivity of the synthesized MBGNs was accessed by immersion in Simulated Body Fluid (SBF), as introduced by Kokubo et al. [46]. The synthesized particles $(20 \mathrm{mg})$ were pressed into pellets, which were immersed in $40 \mathrm{~mL}$ of SBF and incubated at $37^{\circ} \mathrm{C}$ for 7,14 and 21 days. At each time point samples were removed from SBF, rinsed with distilled water and dried in an oven at $60{ }^{\circ} \mathrm{C}$. The dried samples were subsequently characterized using FE-SEM, EDX and XRD to detect the possible formation of hydroxyapatite (HAp) crystals.

\subsection{Antibacterial study of Mn-MBNGs}

Mn-doped bioactive glass pellets were investigated for the antibacterial affect against Bacillus subtilis (B. subtilis) strain 168, Escherichia coli (E. coli) C600, Pseudomonas aeruginosa (P. aeruginosa) PAO1 and Staphylococcus aureus (S. aureus) DSM20231. The bacteria cultures were prepared by inoculating $5 \mathrm{ml}$ of Lura Bertani (LB) medium [47] with each bacterial strain, followed by incubation at $37^{\circ} \mathrm{C}$ for $16 \mathrm{~h} .20 \mu \mathrm{l}$ aliquots of the overnight cultures were transferred to the wells of a sterile 6 well cell culture plate (Greiner, Frickenhausen, Germany) filled with $1980 \mu \mathrm{l}$ of fresh LB medium. Finally, Mn-MBGNs pellets and MBGNs pellets (control sample) were immersed in the well plates. The prepared well plates were incubated for 3 days at $37^{\circ} \mathrm{C}$.

\subsection{Ion release in DMEM}

Ion release characteristics of synthesized Mn-MBGNs, soaked in Dulbecco's Modified Eagle Medium (DMEM; Sigma-Aldrich, Germany), were quantified using an inductively coupled plasma-optical emission spectrometer (ICP-OES, Opteima 8300, Pekrin Elmer). For the ICP-OES analysis 6-point calibrations (100, 50, 25, 10, 5 and $1 \mathrm{ppm})$ 
were performed by diluting certified standards. Samples were measured in triplicate and mean values with standard deviations are reported. Briefly, $60 \mathrm{mg}$ of each type of samples were soaked in $40 \mathrm{ml}$ of DMEM at $37^{\circ} \mathrm{C}$. At each predetermined time point, $5 \mathrm{ml}$ of supernatant liquid was taken out for elemental analysis and diluted with deionized water. The fresh DMEM was refilled to maintain constant the total DMEM volume.

\section{Results}

\subsection{Morphological and structural characteristic of MBGNs}

The morphology of synthesized mesoporous bioactive glass nanoparticles doped with different concentrations of manganese $(0,3,5$ and $7(\mathrm{~mol} \%))$ was observed by FE-SEM, as presented in Fig. 1.

TEM and all other material characterization results (BET, EDX, FTIR, XRD, ICP-OES, in vitro bioactivity and antibacterial activity) are shown only for 5 Mn-MBGNs (Fig. 2) because SEM results (Fig. 1) showed that the morphology of the synthesized mesoporous bioactive glass nanoparticles is affected at higher $\mathrm{Mn}$ content (7 Mn-MBGNs). Moreover, previous studies have also shown that $5 \mathrm{~mol} \% \mathrm{Mn}$ addition to bioactive glass particles does not negatively affect the bioactivity but supports the proliferation of osteoblastic cells $[28,29]$. Thus, we selected 5 Mn-MBGNs as the optimum composition for the further characterization of Mn doped MBGNs. TEM (Fig. 2) and other characterization results (Figs. 3-6) on MBGNs are presented in this section in order to demonstrate the effect of $\mathrm{Mn}$ on the properties of mesoporous bioactive glass nanoparticles.

Energy-dispersive X-ray spectroscopic (EDX) analysis confirmed the incorporation of $\mathrm{P}$ and $\mathrm{Mn}$ in the MBGNs, as shown in Fig. 3, while Fig. 4 shows the EDX mapping analysis of 5 Mn-MBGNs confirming that $\mathrm{Ca}$ (yellow), $\mathrm{Mn}$ (red) and $\mathrm{Si}$ (blue) are uniformly distributed in the nanoparticles.

Figure 5 shows the nitrogen adsorption-desorption isotherms and the pore size distributions of synthesized MBGNs and 5 Mn-MBGNs. The textural properties of samples derived from nitrogen-sorption analysis are displayed in Table 2.

XRD patterns of MBGNs and 5 Mn-MBGNs which were sintered at $700{ }^{\circ} \mathrm{C}$ are shown in Fig. 6. All XRD patterns are quite identical with no significant difference in doped and
Fig. 1 SEM images showing the morphology of the synthesized BG particles: (a) MBGNs, (b) 3 Mn-MBGNs, (c) 5 Mn-MBGNs and (d) 7 Mn-MBGNs
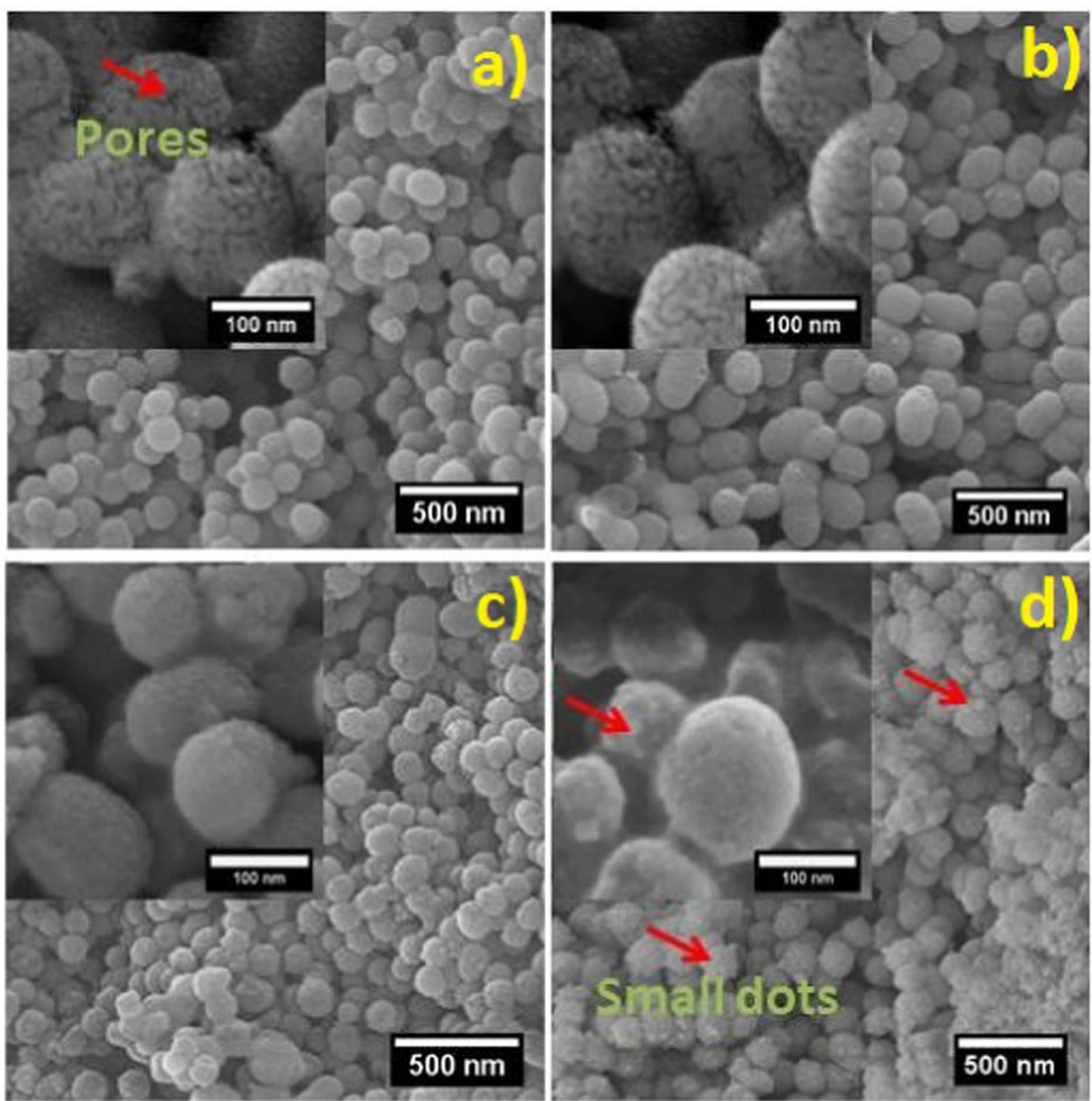
Fig. 2 TEM images of (a) MBGNs and (b) 5 Mn-MBGNs
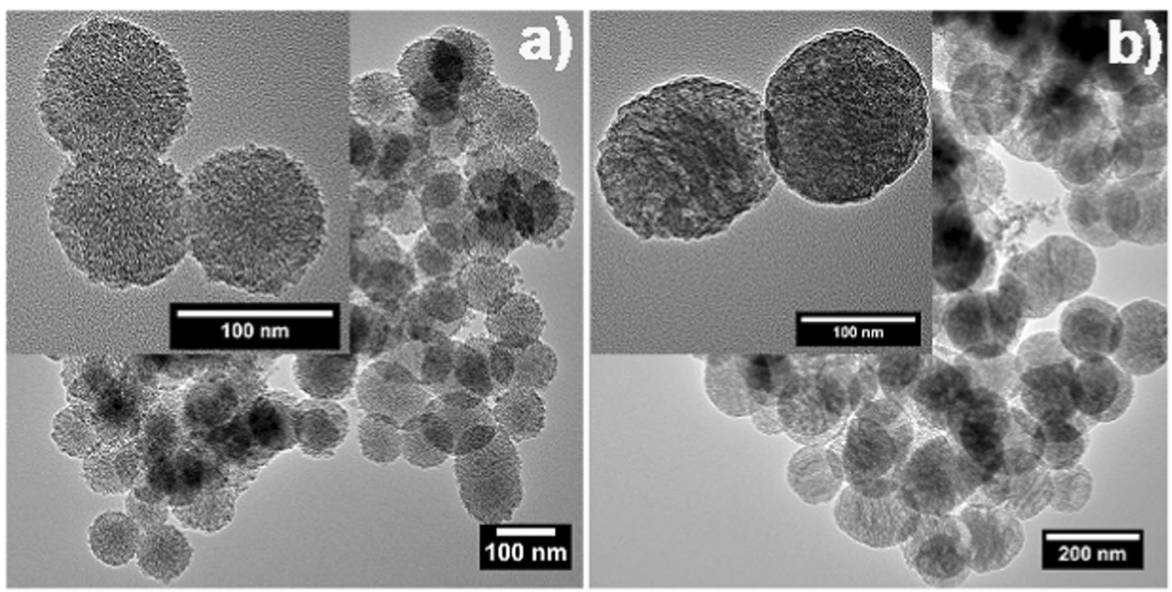

Fig. 3 EDX spectra of the synthesized BG particles (a) MBGNs and (b) 5 Mn-MBGNs
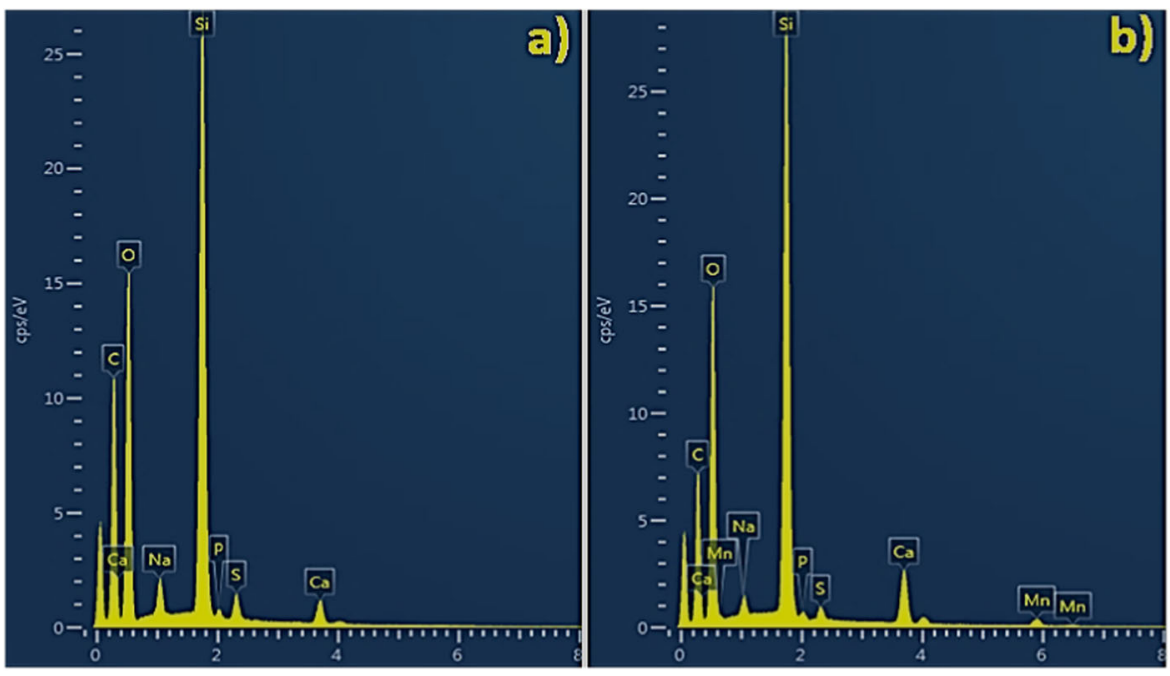

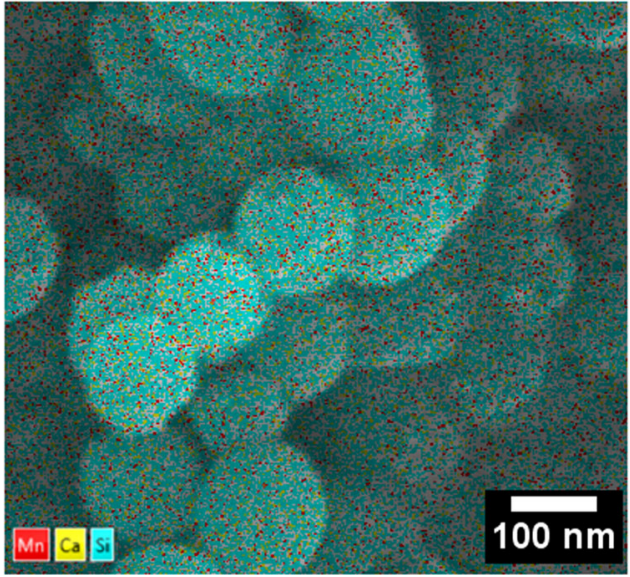

Fig. 4 EDX mapping analysis of 5 Mn-MBGNs showing that $\mathrm{Ca}$ (yellow), Mn (red) and $\mathrm{Si}$ (blued) are uniformly distributed in the nanoparticles

un-doped conditions. A broad band in the range of $20^{\circ}-34^{\circ}$ $(2 \theta)$ was observed, which indicates the amorphous nature of all samples. The molecular structure of the synthesized bioactive glasses and the effect of $\mathrm{Mn}$ on the glass network were studied by FTIR spectroscopy (Fig. 6 - left).

\subsubsection{In vitro bioactivity of Mn-MBNGs}

SEM analysis showed the change in the morphology of particles (MBGNs, 5 Mn-MBGNs) upon immersion in SBF for 7 days (Fig. 7).

EDX spectra demonstrate an increase in the intensity of $\mathrm{P}$ and Ca peaks and a decrease in the intensity of Si peaks of MBGNs and 5 Mn-MBGNs after immersion in SBF (Fig. 8 ), which is related to the deposition of calcium phosphates [48, 49].

Furthermore, XRD results support the formation of hydroxyapatite (HAp) crystals after immersion in SBF (Fig. 9).

\subsubsection{Ion release from nanoparticles in DMEM}

Figure 10 shows the cumulative ion release (measured in $\mathrm{ppm}$ ) of 5 Mn-MBGNs powder, when immersed in DMEM 

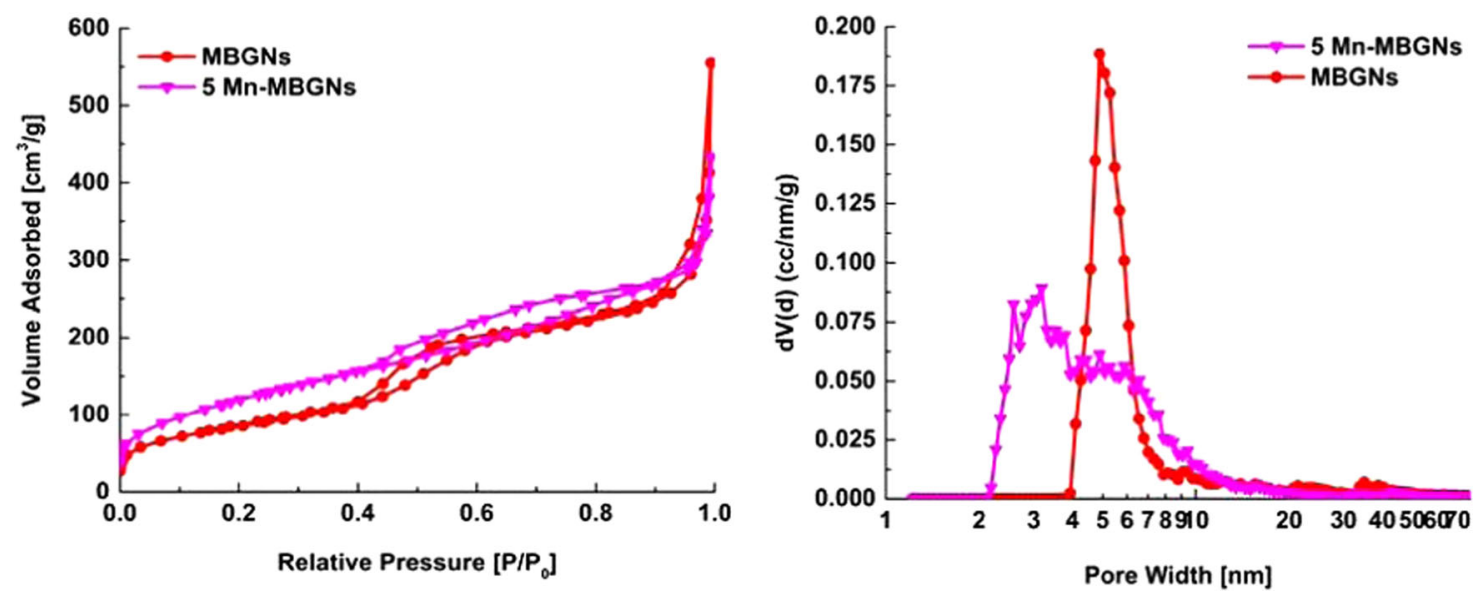

Fig. 5 Nitrogen adsorption-desorption isotherms of synthesized bioactive glass nanoparticles (left) and pore size distribution (right)

Table 2 Textural properties of synthesized BG nanoparticles

\begin{tabular}{llll}
\hline Bioactive glass types & BET surface area $\left(\mathrm{m}^{2} / \mathrm{g}\right)$ & Total pore volume ${ }^{\mathrm{a}}\left(\mathrm{cm}^{3} / \mathrm{g}\right)$ & Average pore diameter $(\mathrm{nm})$ \\
\hline MBGNs & 309 & 0.86 & 11.1 \\
5 Mn-MBGNs & 444 & 0.70 & 6.03 \\
\hline
\end{tabular}

${ }^{\mathrm{a}}$ Total pore volume at $\mathrm{P} / \mathrm{P}_{\mathrm{o}}=0.997$
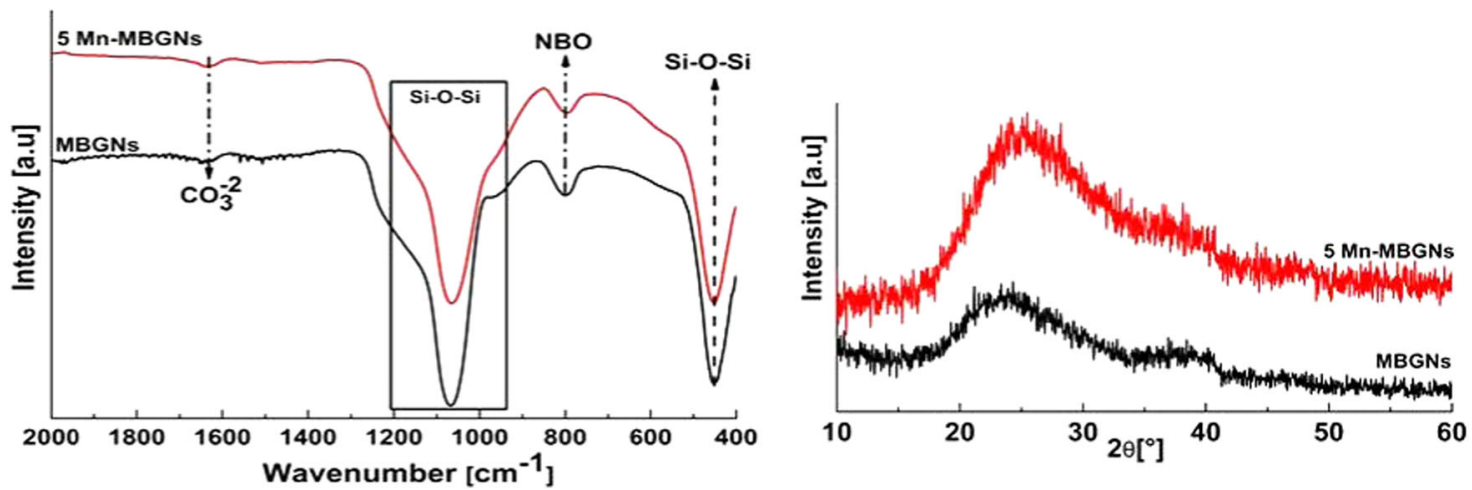

Fig. 6 XRD analysis (right) and FTIR spectra (left) of the synthesized BG nanoparticles doped with various concentrations of Mn

for 15 days. DMEM is used to access the degradation behavior of the materials in a relevant cell culture environment [50]. Generally, the release of $\mathrm{Ca}^{2+}, \mathrm{SiO}_{4}{ }^{-4}, \mathrm{PO}_{4}{ }^{3-}$ and $\mathrm{Mn}^{2+}$ ions increases with increase in soaking time [18].

\subsubsection{Antibacterial activity of BG nanoparticles}

The antibacterial activity of the new BG nanoparticles was investigated against $B$. subtilis and $E$. coli, two widely used model species for Gram-positive and Gram-negative bacteria, as well as of $P$. aeruginosa and $S$. aureus, two human pathogens frequently found connected to hospital infections. Results are shown in Fig. 11. The optical density of cultures was measured at $600 \mathrm{~nm}\left(\mathrm{OD}_{600}\right)$. MBGN showed antibacterial activity in LB medium in order of $P$. aeruginosa $>$ E. coli $>$ B. subtilis $>$ S. aureus, as shown in Table 3.

\section{Discussion}

\subsection{Bioactive glass nanoparticle morphology and composition}

SEM images (Fig. 1) of the synthesized mesoporous bioactive glass nanoparticles showed the effect of $\mathrm{Mn}$ addition at various concentrations. It was observed that the addition of Mn precursors above the threshold value ( $5 \mathrm{~mol}$ $\% \mathrm{Mn})$ affected the morphology of particles. For instance, 
Fig. 7 SEM images showing the change in the morphology of particles after immersion in SBF: (a) MBGNs after 7 days, (b) MBGNs after 21 days, (c) 5 Mn-MBGNs after 7 days, (d) 5 Mn-MBGNs after 21 days
Fig. 8 EDX analysis showing the change in elemental composition of $\mathrm{Ca}, \mathrm{Si}$ and $\mathrm{P}$ after immersion in SBF: (a) MBGNs after 21 days, (b) 5 MnMBGNs after 21 days
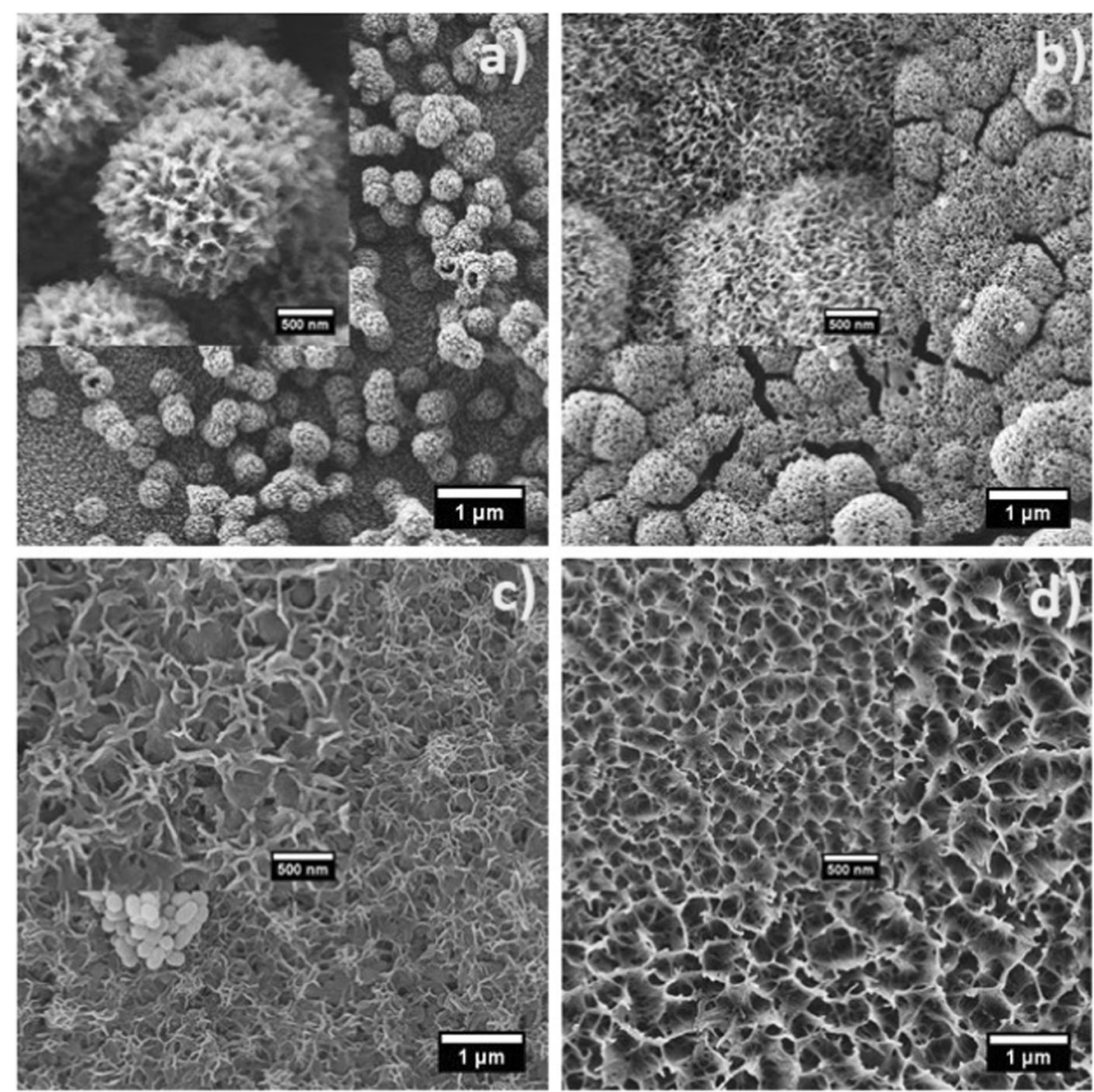
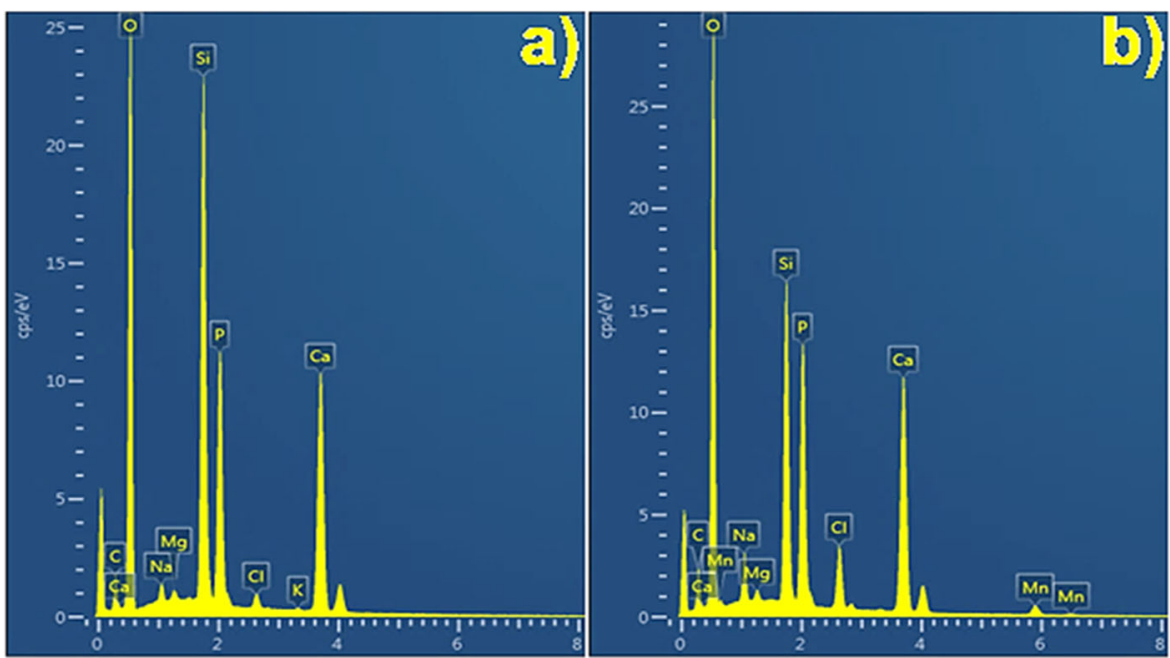

MBGNs showed a smooth surface in contrast to $7 \mathrm{Mn}$ MBGNs, which have relatively rough surfaces exhibiting small dots that could be due to adsorbed silica particles on the surface (Fig. 1d) [51]. It has been reported that a relatively high amount of metallic ions during synthesis affects condensation and shifts the growth equilibrium, which may lead to an increase of the surface roughness of particles
$[52,53]$. Thus, we conclude that the synthesized MBGNs (doped and un-doped) exhibited the typical characteristics (shape, uniformity and dispersion) of silicate particles synthesized by the Stöber method [54].

Manganese-doped bioactive glass nanoparticles (Mn-MBGNs) developed in this study are uniform, well dispersed and show a spheroidal morphology with particle 

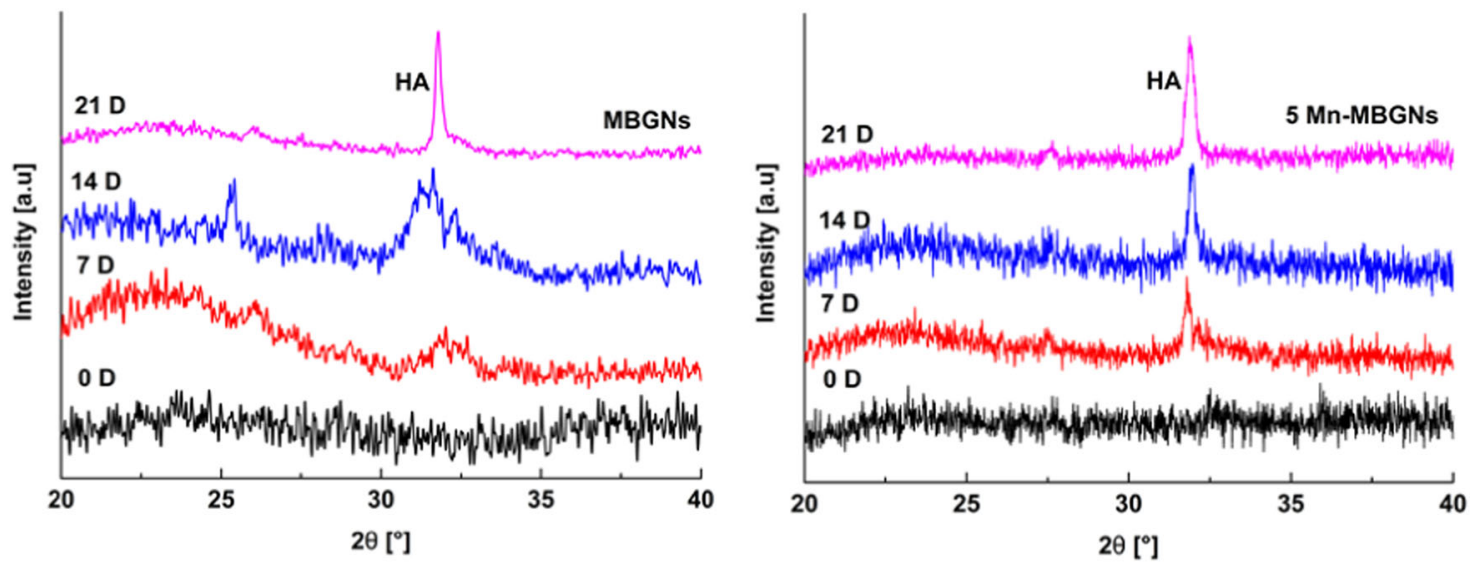

Fig. 9 XRD patterns before and after treatment in SBF for 7, 14 and 21 days of MBGNs (left) and 5 Mn-MBGNs (right)
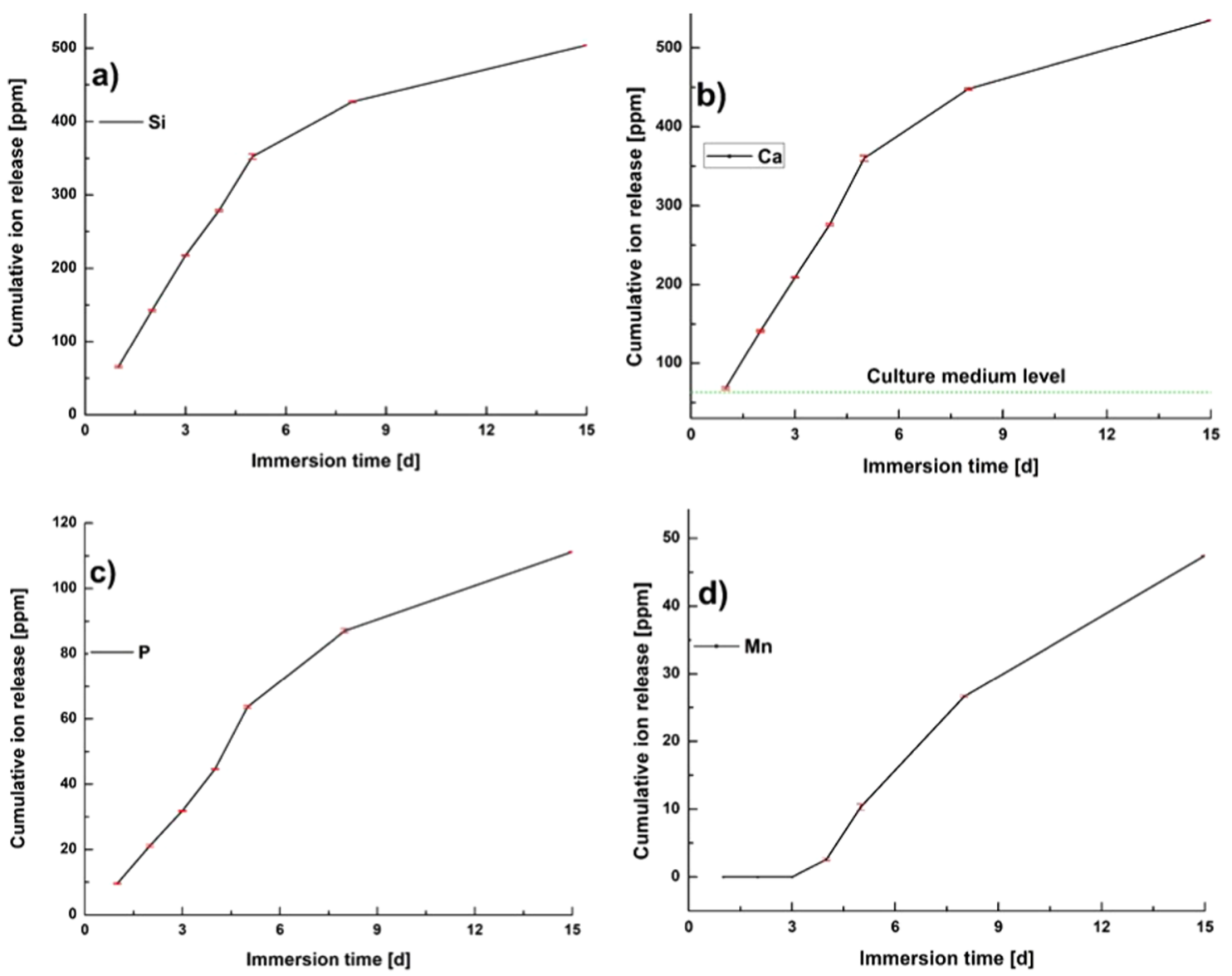

Fig. 10 Cumulative ion release profile of 5 Mn-MBGNs in DMEM for 15 days. $\mathrm{Ca}, \mathrm{Si}$ and $\mathrm{P}$ ions show an initial burst release followed by slow release. Initially up to three days no Mn release was observed and

after that a sustained release can be noticed (S.D. was obtained from three samples)

sizes in the range $(110 \pm 10) \mathrm{nm}$ (Fig. 1). The particle size was measured by the "Image J" software by measuring 100 particles (observed in SEM images, Fig. 1) for each prepared bioactive glass composition. The surface properties of the particles play an important role in agglomeration. High surface area to volume ratio of nanoparticles provides a very

high total surface energy of the system, which can be reduced e.g., by ripening, adsorption, aggregation or agglomeration, i.e., the formation of larger structures. Moreover, formation of agglomerates is due to the strong van der Waals interaction forces between the particles [55]. The particle size of MBGNs and 5 Mn-MBGNs was fairly 


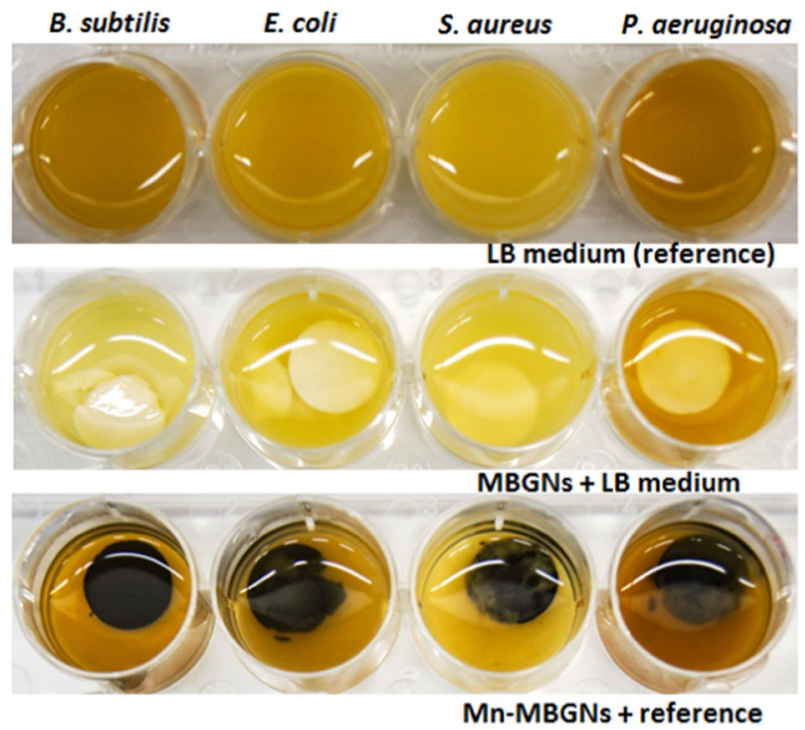

Fig. 11 Antibacterial susceptibility test of synthesized MBGNs and 5 Mn-MBGNs pellets against different bacterial strains, which shows a decrease in turbidity of the growth medium after immersion the pellets in the bacteria culture 6-well plates. The pellets are incubated for 3 days at $37^{\circ} \mathrm{C}$

Table 3 Antibacterial susceptibility test showing a relative decrease in the $\mathrm{OD}_{600}$ after immersion of MBGNs pellets in the bacteria culture well plates

\begin{tabular}{lll}
\hline Species & $\begin{array}{l}\text { LB without MBGN } \\
\text { addition }\end{array}$ & $\begin{array}{l}\text { LB with added MBGN } \\
\text { pellet }\end{array}$ \\
\hline B. subtilis & 5.0 & 2.5 \\
E. coli & 7.0 & 0.5 \\
P. aeruginosa & 14.5 & 0.1 \\
S. aureus & 7.0 & 4.5 \\
\hline
\end{tabular}

Experiments were carried out in duplicate with similar results

similar, which could be due to the same valence state of $\mathrm{Ca}$ and Mn. Recently, Barrioni et al. [29] described that Mn replaces calcium in the glass network due to the same valence state $(+2)$. A similar effect (substitution of $\mathrm{Ca}$ with $\mathrm{Mn}$ ) is hypothesized to occur in this study. However, Mn can also exist as Mn (III), Mn (IV), and Mn (VII) but in that case substitution of Mn with calcium would be less likely. Mn (II) is expected to be most stable under the moderate conditions / aqueous environment under which the particles were synthesized. Indeed further studies to determine the valence state of $\mathrm{Mn}$ in the glass network are required, this being however beyond the scope of the present investigation.

As indicated above, on the basis of the SEM results (Fig.1) we chose MBGNs and 5 Mn-MBGNs for TEM analysis. It was shown (Fig. 2a) that MBGNs particles have spherical morphology with average particle size of $110 \pm 10$ $\mathrm{nm}$ (particle size was measured by "Image J"). It was possible to observe on high magnification TEM images that MBGNs particles have disordered porous structure (inset of Fig. 2a). Figure $2 \mathrm{~b}$ confirmed that $5 \mathrm{Mn}-\mathrm{MGBNs}$ particles also exhibit spherical morphology with average particle size of $100 \pm 10 \mathrm{~nm}$. The particle sizes of MBGNs and $5 \mathrm{Mn}$ MBGNs were statistically similar. Moreover, textural properties of MBGNs and 5 Mn-MBGNs derived from nitrogen-sorption analysis indicated IUPAC Type IV isotherms, which confirmed the presence of mesoporosity. Moreover, high nitrogen uptake is observed at $\mathrm{P} / \mathrm{Po} \approx 0.99$, which indicates that the samples exhibit nano to submicronsized particles. MBGNs exhibit a sharp nitrogen uptake at relative pressure $(\mathrm{P} / \mathrm{Po})$ of $0.40-0.50$ that corresponds to the presence of medium size mesopores $(4-10 \mathrm{~nm})$. The average pore size decreases by incorporating Mn (also reported in [29]), as illustrated in Table 2. Nevertheless, addition of manganese led to the formation of small, medium and large size mesopores resulting in the increased BET surface area (Table 2). TEM images (Fig. 2) and BET results (Table 2) confirmed the mesoporous structure of the particles, which is relevant in relation to their in vitro bioactivity (higher surface porosity leads to high surface area [56]). Furthermore, porous structures provide potential for loading drugs or biomolecules, which becomes important when considering applications of MBGNs as drug delivery carriers $[57,58]$.

In the context of the morphological studies of the synthesized mesoporous bioactive glass nanoparticles, we performed a qualitative compositional analysis by EDX on MBGNs and 5 Mn-MBGNs (Fig. 3). Ca, P, Si peaks in the EDX spectrum confirm their presence in MBGNs particles (Fig. 3A). Figure $3 \mathrm{~b}$ indicates the presence of a Mn peak in addition to the $\mathrm{Ca}, \mathrm{P}$ and $\mathrm{Si}$ peaks, which corresponds to the addition of $\mathrm{Mn}$ in MBGNs. The calculated molar ratio $(\mathrm{Ca} /$ $\mathrm{Si}(0.1288)+\mathrm{Mn} / \mathrm{Si}(0.0080))$ in $5 \mathrm{Mn}-\mathrm{MBGN}$ s was close to the $\mathrm{Ca} / \mathrm{Si}(0.1302)$ ratio of MBGNs, which indicates that Mn has been incorporated in the silicate network. A similar approach has been used to determine the incorporation of copper in the silicate network by Bari et al. [53]. Furthermore, the incorporation of $\mathrm{Mn}$ in the silica network has been shown by XRD analysis by Barrioni, et al. [29] who did not observe any peak related to the $\mathrm{MnCl}_{2}$ precursor. It is clear that further studies such as NMR spectroscopy measurements will be helpful to determine precisely the role of $\mathrm{Mn}$ in the glass network. Furthermore, EDX mapping investigated the distribution of elements in 5 Mn-MBGNs, which showed that $\mathrm{Mn}, \mathrm{Ca}, \mathrm{Si}$ were evenly distributed in the particles (Fig. 4).

After acquiring conclusive evidence about the presence of $\mathrm{Mn}$ in the synthesized MBGNs, XRD analysis revealed the amorphous structure of MBGNs and 5 Mn-MBGNs (Fig. 6), represented by the broad halo in the range of $20^{\circ}-34^{\circ}(2 \theta)$. Moreover, XRD patterns did not show any 
crystalline peak related to the Mn reagent, which may indicate that $\mathrm{Mn}$ has been incorporated in the glass structure. BGs having amorphous structure exhibit better dissolution rates, which results in higher bioactivity compared to BGs containing crystalline phases $[29,59]$.

The molecular structure of the synthesized bioactive glasses and the effect of $\mathrm{Mn}$ on the glass network were studied by FTIR spectroscopy (Fig. 6). The presence of bands at $455 \mathrm{~cm}^{-1}$ and $1067 \mathrm{~cm}^{-1}$ can be assigned to $\mathrm{Si}-\mathrm{O}-\mathrm{Si}$ stretching and $\mathrm{Si}-\mathrm{O}-\mathrm{Si}$ bending modes, respectively [60]. FTIR spectra show the peak at $1635 \mathrm{~cm}^{-1}$, which is ascribed to the carbonate band in MBGNs. Nevertheless, the intensity of non-bridging oxygen (NBO) peak in the silica network (observed at $799 \mathrm{~cm}^{-1}$ for MBGNs $[18,44]$ ) decreased relatively with the addition of Mn (Fig. 6), which could be due to the bonding between unbonded oxygen (in the silica network) and Mn. However, the reduction in the intensity of NBO with the addition of Mn cannot be considered as an evidence of Mn acting as network modifier.

\subsection{Bioactivity in SBF}

The formation of hydroxyapatite (HA) crystals on the surface of biomaterials upon immersion in SBF is the qualitative indication of the bone bonding ability of materials, particularly bioactive glasses and ceramics [5-7, 48]. The rate of formation of HA crystals on BGs in SBF depends on several factors such as; glass composition, surface topography and dissolution rate [29, 61, 62]. In this study, the bioactivity of MBGNs and 5 Mn-MBGNs was assessed. SEM analysis showed the change in the morphology of particles (MBGNs, 5 Mn-MBGNs) upon immersion in SBF for 7 days (Fig. 7). The typical porous structure of apatite crystals was observed on the particles [48, 63]. Moreover, with the increase in immersion time plate like crystals were seen to grow on the surface of the 5 Mn-MBGNs, as shown in Fig. 7c, d. The plate like morphology suggests the formation of calcium enriched apatite $[48,49]$. The $\mathrm{pH}$ of SBF is 7.40 which is above the isoelectric point of the particles. This means that the surface charge of the particles was negative when immersed in $\mathrm{SBF}$, which may have led to the adsorption of $\mathrm{Ca}^{2+}$ ions from the SBF solution and eventually to the formation of a crystalline layer of calcium enriched hydroxyapatite, as shown in Fig. 7c, d (described in refs. [48, 64]). However, MBGNs showed a needle like morphology in the high magnification images, as shown in Fig. 7a, b. Such needle like crystals indicate the formation calcium deficient apatite. The reason could be the fact that MBGNs are relatively less negatively charged at $\mathrm{pH}$ of 7.40 . Thus, leading to less adsorption of $\mathrm{Ca}^{2+}$ ions from the SBF solution and eventually to the formation of a crystalline layer of calcium deficient hydroxyapatite, as shown in Fig. 7a, b (as described in refs. [49, 63]).

EDX spectra demonstrated an increase in the intensity of $\mathrm{P}$ and $\mathrm{Ca}$ peaks and a decrease in the intensity of Si peaks of MBGNs and 5 Mn-MBGNs after immersion in SBF (Fig. 8), which is related to the deposition of calcium phosphates $[48,49]$. Furthermore, XRD results support the formation of hydroxyapatite (HAp) crystals after immersion in SBF. XRD patterns for MBGNs and 5 Mn-MBGNs (Fig. 9) show a semi crystalline phase with the main peak at $32^{\circ}$, after 7 days of immersion in SBF. The crystalline pattern of the new phase matches with the XRD pattern of hydroxyapatite (JCPDS 72-1243). Moreover, the relative intensity of the peak at $32^{\circ}$ increases with increase in immersion time for both types of glasses, as shown in Fig. 9. In general, the EDX and XRD results are compatible with previous results of Zheng et al. [18], who reported the in vitro bioactivity of monodispersed $\mathrm{Cu}$ doped bioactive glass nanoparticles in simulated body fluid (SBF). It can be concluded on the basis of bioactivity results in SBF that the release of Mn ions (illustrated by the ICP-OES results) after 7 days improves the bioactivity of the synthesized bioactive glass particles. The reason for the improved bioactivity associated with the 5 Mn-MBGNs is likely the higher dissolution rate of the particles in comparison to MBGNs [56]. This increase in the dissolution rate could be associated with the increased surface area (decrease in pore size), as shown by the BET results (Table 2). However, the relatively slow mineralization kinetics of MBGNs can be due to the relatively low surface area of these particles in comparison to that of 5 Mn-MBGNs. Nevertheless, long term in vitro bioactivity and in vivo studies should be done to verify the behavior of Mn-doped bioactive glasses in more relevant physiological conditions.

Figure 10 shows the cumulative ion release (measured in ppm) of 5 Mn-MBGNs powder, when immersed in DMEM for 15 days. DMEM is used to access the degradation behavior of the materials in a relevant cell culture environment [50]. DMEM contains approx. $62 \mathrm{ppm}$ of $\mathrm{Ca}$ ions [50] which is subtracted from the release profile of $\mathrm{Ca}$, as shown in Fig. 10d. The error bars in Fig. 10 indicate the standard deviation of the respective concentrations, which was calculated from three single determinations. $\mathrm{Si}$ and $\mathrm{Ca}$ ions show a rapid release in the first 5 days, followed by a relatively slow release up to 15 days. The phosphorous $(\mathrm{P})$ ion concentration also increased with soaking time. However, the cumulative release of $\mathrm{P}$ species is lesser in comparison to that of $\mathrm{Si}$ and $\mathrm{Ca}$, due to the lower amount of $\mathrm{P}$ present in the synthesized bioactive glass. Initially for 3 days, no release of Mn ions could be detected; afterwards, $\mathrm{Mn}$ ions were released slowly with the cumulative release of 47 ppm after 15 days. A Mn-depleted surface layer likely caused low Mn levels for 1d/3d immersion times which 
were not detectable. In another study, $5 \%$ Mn doped bioactive glass (sol-gel synthesized) showed cumulative ionic release of $\mathrm{Si}, \mathrm{P}, \mathrm{Ca}$ and $\mathrm{Mn}: 360,83,850$ and $11 \mathrm{ppm}$, respectively [29]. However slight variations in the ion release concentration have been observed among different researchers, as well as in the current study, possibly due to differences in the synthesis methods and elements incorporated in the glass network.

On the basis of the ICP-OES results $\mathrm{Mn}$ ion release was confirmed to occur in a controlled and sustained manner. It is important to highlight that the release of $\mathrm{Si}$ and $\mathrm{Ca}$ ions indicates that the synthesized bioactive glasses are degradable, which is relevant for bone healing and regeneration. Furthermore, the release of biological active ions is expected to improve the particles bioactivity $[6,7]$.

Generally, the release of $\mathrm{Ca}^{2+}, \mathrm{SiO}_{4}{ }^{-4}, \mathrm{PO}_{4}{ }^{3-}$ and $\mathrm{Mn}^{2+}$ ions increases with increase in soaking time [18]. Hence the data suggest that the bioactive glass chemistry can be adjusted to release an appropriate amount of different ions such as $\mathrm{Mn}, \mathrm{Si}, \mathrm{Ca}$ and $\mathrm{P}$, depending on the applications. However, a long-term Mn release study should be done in order to investigate in detail the release kinetics of $\mathrm{Mn}$ doped bioactive glasses in realistic conditions.

It is important to highlight that the ion release study was done in DMEM in contrast to the bioactivity study, which was carried out in SBF. The reason was that DMEM can access the degradability of materials in more relevant physiological conditions because it contains potassium, calcium, and amino acids (SBF contains the ionic concentration of human blood plasma but it does not contain proteins $[18,43])$.

\subsection{Antibacterial activity}

To test the antibacterial activity of BGs, growth of $B$. subtilis and E. coli, two widely used model species for Gram-positive and Gram-negative bacteria, as well as of $P$. aeruginosa and $S$. aureus, two human pathogens frequently found connected to nosocomial (hospital-acquired) infections, was studied. When the growth of the different bacteria was tested, compared to the reference wells without pellets, MBGNs and $5 \mathrm{Mn}-\mathrm{MBGNs}$ were found to reduce growth, as indicated by the decreased turbidity of the growth medium (Fig. 11). As a quantitative approach, the optical density of cultures was measured at $600 \mathrm{~nm}\left(\mathrm{OD}_{600}\right)$. These $\mathrm{OD}_{600}$ measurements revealed that growth of bacteria in LB medium decreased with the addition of MBGNs. Quantitatively, MBGN showed antibacterial activity in LB medium in order of $P$. aeruginosa $>$ E. coli $>$ B. subtilis $>S$. aureus, as shown in Table 3. Taken together, the results indicate that MBGNs are active against bacteria including important human pathogens. The optical density of bacterial cultures with added 5 Mn-MBGNs could not be measured due to the fact that the medium stained black over time because of the inherent color of the synthesized Mn-doped mesoporous bioactive glass nanoparticles released into the medium [59, 61, 62]. This effect was absent in case of MBGNs, which did not change the color of the medium due to the absence of Mn. 45S5 BG has been reported to exhibit antibacterial effect $[49,63]$, but there are only few studies on the antibacterial effect of sol gel bioactive glasses [65, 66]. The antibacterial activity of bioactive glass is usually associated with the $\mathrm{pH}$ increase due to BG dissolution [48, 49]. This study will pave the way for future investigations on the antibacterial activity of Mn doped BGs synthesized via sol-gel method. Furthermore, the slow release of $\mathrm{Mn}$ may provide long term antibacterial activity to MBGNs, which is beneficial for wound healing efficiency. The therapeutic limit for $\mathrm{Mn}$ ions is reported to be $5.49 \mathrm{ppm}$ [29]. However, while Mn is known for its beneficial effect on integrin affinity [67], the range of $\mathrm{Mn}$ ion concentration that can be toxic for cells has not been clearly identified in literature. Our preliminary cell viability study (WST-8) on 5 Mn-MBGNs revealed a non-toxic behavior of this BG towards MG-63 cells (data not shown here).

\section{Conclusions}

In this study Mn-MBGNs particles were synthesized by a modified Stöber method. SEM and TEM observations showed that the synthesized Mn-MBGNs have a spheroidal morphology and disordered mesoporous structure. EDX and XRD analyses confirmed that Mn has been doped in the silica network. BET results elucidated that synthesized MBGNs exhibit high BET surface area and mesoporosity. However, the average pore size decreases with the increase in $\mathrm{Mn}$ content. Mn-doped bioactive glass particles form apatite crystals upon immersion in simulate body fluid. Elemental analysis by ICP-OES indicated a relatively slow release of Mn ions, which suggests the possibility of using Mn-MBGNs as vehicles for delivering therapeutic Mn ions. BGNs and Mn-MBGNs exhibited antibacterial activity against B. subtilis, E. coli, P. aeruginosa and S. aureus. Future work will focus on investigating possible long-term cytotoxic effects of Mn-MBGNs and on developing different drug loading strategies for these novel MBGNs.

Acknowledgements QN would like to thank Higher Education Commission (HEC) of Pakistan for granting a scholarship. Authors would like to thank the TEM facilities of CITIUS-US. TEM analyses have been funded by Grant P2017/837 from the University of Seville (Spain). 


\section{Compliance with ethical standards}

Conflict of interest The authors declare that they have no conflict of interest.

\section{References}

1. Nicolini V, Malavasi G, Menabue L, Lusvardi G, Benedetti F, Valeri S, Luches P. Cerium-doped bioactive 45S5 glasses: spectroscopic, redox, bioactivity and biocatalytic properties. J Mater Sci. 2017;52:8845-57.

2. Eqtesadi S, Motealleh A, Miranda P, Pajares A, Lemos A, Ferreira JMF. Robocasting of 45S5 bioactive glass scaffolds for bone tissue engineering. J Eur Ceram Soc. 2014;34:107-18.

3. Gerhardt L-C, Boccaccini AR. Bioactive Glass and Glass-Ceramic Scaffolds for Bone Tissue Engineering. Materials. 2010;3:3867-910.

4. Salgado AJ, Coutinho OP, Reis RL. Bone tissue engineering: State of the art and future trends. Macromol Biosci. 2004;4:743-65.

5. Hench LL, hompson I. Twenty-first century challenges for biomaterials. J R Soc Interface. 2014;7:S379-91.

6. Hench LL. The story of Bioglass. J Mater Sci Mater Med. 2006;17:967-78.

7. Jones JR, Review of bioactive glass: From Hench to hybrids. Acta Biomater. 2015;23:S53-82.

8. Miguez-Pacheco V, Hench LL, Boccaccini AR. Bioactive glasses beyond bone and teeth: Emerging applications in contact with soft tissues. Acta Biomater. 2015;13:1-15.

9. Schröder HC, Wang XH, Wiens M, Diehl-Seifert B, Kropf K, Schloßmacher U, Müller WEG, Silicate modulates the cross-talk between osteoblasts (SaOS-2) and osteoclasts (RAW 264.7 cells): Inhibition of osteoclast gro wth and differentiation. J Cell Biochem. 2012;113:3197-206.

10. Shie MY, Ding SJ, Chang HC. The role of silicon in osteoblastlike cell proliferation and apoptosis. Acta Biomater. 2011;7:2604-14.

11. Haro Durand LA, Vargas GE, Romero NM, Vera-Mesones R, Porto-López JM, Boccaccini AR, Zago MP, Baldi A, Gorustovich A. Angiogenic effects of ionic dissolution products released from a boron-doped 45S5 bioactive glass. J Mater Chem B. 2015;3:1142-8.

12. Balasubramanian P, Hupa L, Jokic B, Detsch R, Grünewald A, Boccaccini AR, Angiogenic potential of boron-containing bioactive glasses: in vitro study. J Mater Sci. 2017;52:8785-92.

13. Hench LL, Chronology of Bioactive Glass Development and Clinical Applications. New J Glas Ceram. 2013;3:67-73.

14. Qazi TH, Hafeez S, Schmidt J, Duda GN, Boccaccini AR, Lippens E. Comparison of the effects of 45S5 and 1393 bioactive glass microparticles on hMSC behavior. J Biomed Mater Res-Part A. $2017 ; 105: 2772-82$.

15. Hoppe A, Guldal NS, Boccaccini AR. A review of the biological response to ionic dissolution products from bioactive glasses and glass-ceramics. Biomaterials. 2011;32:2757-74.

16. Salinas AJ, Shruti S, Malavasi G, Menabue L, Vallet-Regí M. Substitutions of cerium, gallium and zinc in ordered mesoporous bioactive glasses. Acta Biomater. 2011;7:3452-8.

17. Wu C, Zhou Y, Fan W, Han P, Chang J, Yuen J, Zhang M, Xiao Y. Hypoxia-mimicking mesoporous bioactive glass scaffolds with controllable cobalt ion release for bone tissue engineering. Biomaterials. 2012;33:2076-85.

18. Zheng K, Dai X, Lu M, Huser N, Taccardi N, Boccaccini AR. Synthesis of copper-containing bioactive glass nanoparticles using a modified Stober method for biomedical applications. Colloids Surf B Biointerfaces. 2017;150:159-67.

19. Wu C, Zhou Y, Lin C, Chang J, Xiao Y. Strontium-containing mesoporous bioactive glass scaffolds with improved osteogenic/ cementogenic differentiation of periodontal ligament cells for periodontal tissue engineering. Acta Biomater. 2012;8:3805-15.

20. Kavitha RJ, Subha B, Shanmugam S, Ravichandran K. Synthesis and Invitro Characterisation of Lithium Doped Bioactive Glass through Quick Alkali Sol - Gel Method. Int J Innov Res Sci Eng. 2014;2:2347-3207.

21. Miguez-Pacheco V, de Ligny D, Schmidt J, Detsch R, Boccaccini AR. Development and characterization of niobium-releasing silicate bioactive glasses for tissue engineering applications. J Eur Ceram Soc. 2017;38:871-76.

22. Wu C, Miron R, Sculean A, Kaskel S, Doert T, Schulze R, Zhang Y. Proliferation, differentiation and gene expression of osteoblasts in boron-containing associated with dexamethasone deliver from mesoporous bioactive glass scaffolds. Biomaterials. 2011;32:7068-78.

23. Balasubramanian P, Strobel LA, Kneser U, Boccaccini AR. Zinccontaining bioactive glasses for bone regeneration, dental and orthopedic applications. Biomed Glas. 2015;1:51-69.

24. Sopyan I, Ramesh S, Nawawi NA, Tampieri A, Sprio S. Effects of manganese doping on properties of sol-gel derived biphasic calcium phosphate ceramics. Ceram Int. 2011;37:3703-15.

25. Lüthen F, Bulnheim U, Müller PD, Rychly J, Jesswein H, Nebe JGB. Influence of manganese ions on cellular behavior of human osteoblasts in vitro. Biomol Eng. 2007;24:531-6.

26. Srivastava AK, Pyare R, Singh SP. In vitro bioactivity and physical-mechanical properties of $\mathrm{MnO} 2$ substituted 45S5 bioactive glasses and glass-ceramics. J Biomater Tissue Eng. 2012;2:249-58.

27. Fujitani W, Hamada Y, Kawaguchi N, Mori S, Daito K, Uchinaka A, Matsumoto T, Kojima Y, Daito M, Nakano T, Matsuura N. Synthesis of Hydroxyapatite Contining Manganese and Its Evaluation of Biocompatibility. Nano Biomed. 2010;2:37-46.

28. Miola M, Brovarone CV, Maina G, Rossi F, Bergandi L, Ghigo D, Saracino S, Maggiora M, Canuto RA, Muzio G, Vernè E. In vitro study of manganese-doped bioactive glasses for bone regeneration. Mater Sci Eng C. 2014;38:107-18.

29. Barrioni BR, Oliveira AC, de Fatima Leite M, de Magalhaes Pereira M. Sol-gel-derived manganese-releasing bioactive glass as a therapeutic approach for bone tissue engineering. J Mater Sci. 2017;52:8904-27.

30. Creaven BS, Egan DA, Karcz D, Kavanagh K, McCann M, Mahon M, Noble A, Thati B, Walsh M. Synthesis, characterisation and antimicrobial activity of copper(II) and manganese(II) complexes of coumarin-6,7-dioxyacetic acid (cdoaH2) and 4methylcoumarin-6,7-dioxyacetic acid (4-MecdoaH2): X-ray crystal structures of [Cu(cdoa)(phen)2] $8.8 \mathrm{H} 2 \mathrm{O}$ and [Cu(4-Mecdoa) (phen)2] $\cdot 13 \mathrm{H} 2 \mathrm{O}$ (phen $=1,10$-phenanthroline). J Inorg Biochem. 2007;101:1108-19.

31. Sharma N, Jandaik S, Kumar S, Chitkara M, Sandhu IS. Synthesis, characterisation and antimicrobial activity of manganese- and iron-doped zinc oxide nanoparticles. J Exp Nanosci. 2016;11:54-71.

32. Dorkov P, Pantcheva IN, Sheldrick WS, Mayer-Figge H, Petrova R. Mitewa M. Synthesis, structure and antimicrobial activity of manganese(II) and cobalt(II) complexes of the polyether ionophore antibiotic Sodium Monensin A. J Inorg Biochem. 2008;102:26-32.

33. Philippart A, Gómez-Cerezo N, Arcos D, Salinas AJ, Boccardi E, Vallet-Regi M, Boccaccini AR. Novel ion-doped mesoporous glasses for bone tissue engineering: Study of their structural characteristics influenced by the presence of phosphorous oxide. J Non Cryst Solids. 2017;455:90-7. 
34. Erol-Taygun M, Zheng K, Boccaccini AR. Nanoscale Bioactive Glasses in Medical Applications. Int J Appl Glas Sci. 2013;4:136-48.

35. Zhao L, Yan X, Zhou X, Zhou L, Wang H, Tang J, Yu C. Mesoporous bioactive glasses for controlled drug release. Microporous Mesoporous Mater. 2008;109:210-5.

36. Yan X, Yu C, Zhou X, Tang J, Zhao D. Highly ordered mesoporous bioactive glasses with superior in vitro bone-forming bioactivities. Angew Chem-Int Ed. 2004;43:5980-4.

37. Tang F, Li L, Chen D. Mesoporous silica nanoparticles: Synthesis, biocompatibility and drug delivery. Adv Mater. 2012;24:1504-34.

38. Xia W, Chang J. Well-ordered mesoporous bioactive glasses (MBG): A promising bioactive drug delivery system. J Control Release. 2006;110:522-30.

39. Gargiulo N, Cusano AM, Causa F, Caputo D, Netti PA. Silvercontaining mesoporous bioactive glass with improved antibacterial properties. J Mater Sci Mater Med. 2013;24:2129-35.

40. Wu C, Zhou Y, Xu M, Han P, Chen L, Chang J, Xiao Y. Coppercontaining mesoporous bioactive glass scaffolds with multifunctional properties of angiogenesis capacity, osteostimulation and antibacterial activity. Biomaterials. 2013;34:422-33.

41. Zhu Y, Li X, Yang J, Wang S, Gao H, Hanagata N. Compositionstructure-property relationships of the $\mathrm{CaO}-\mathrm{MxOy}-\mathrm{SiO} 2-\mathrm{P} 2 \mathrm{O} 5(\mathrm{M}$ $=\mathrm{Zr}, \mathrm{Mg}, \mathrm{Sr}$ ) mesoporous bioactive glass (MBG) scaffolds. J Mater Chem. 2011;21:9208-18.

42. Wu C, Chang J. Multifunctional mesoporous bioactive glasses for effective delivery of therapeutic ions and drug/growth factors. J Control Release. 2014;193:282-95.

43. Zheng K, Taccardi N, Beltrán AM, Sui B, Zhou T, Marthala VRR, Hartmann M, Boccaccini AR. Timing of calcium nitrate addition affects morphology, dispersity and composition of bioactive glass nanoparticles. RSC Adv. 2016;6:95101-11.

44. Kozon D, Zheng K, Boccardi E, Liu Y, Liverani L, Boccaccini AR. Synthesis of monodispersed Ag-doped bioactive glass nanoparticles via surface modification. Materials. 2016;9:225-32.

45. Ye J, He J, Wang C, Yao K, Gou Z. Copper-containing mesoporous bioactive glass coatings on orbital implants for improving drug delivery capacity and antibacterial activity. Biotechnol Lett. 2014;36:961-8

46. Kokubo T, Takadama H. How useful is SBF in predicting in vivo bone bioactivity? Biomaterials 2006;27:2907-15.

47. Wumes SE, Smith JA, Struhl K. Culinary Biol. 1990;61:17-18.

48. Ur Rehman MA, Ferraris S, Goldmann WH, Perero S, Bastan FE, Nawaz Q, Di Confiengo GG, Ferraris M, Boccaccini AR. Antibacterial and Bioactive Coatings Based on RF Co-sputtering of Silver Nanocluster-silica Coatings on PEEK/ bioactive Glass Layers Obtained by Electrophoretic Deposition. ACS Appl Mater Interfaces. 2017;9:32489-97.

49. Pishbin F, Mouriño V, Gilchrist JB, McComb DW, Kreppel S, Salih V, Ryan MP, Boccaccini AR. Single-step electrochemical deposition of antimicrobial orthopaedic coatings based on a bioactive glass/chitosan/nano-silver composite system. Acta Biomater. 2013;9:7469-79.

50. Stähli C, James-Bhasin M, Hoppe A, Boccaccini AR, Nazhat SN. Effect of ion release from Cu-doped 45S5 Bioglass on 3D endothelial cell morphogenesis. Acta Biomater. 2015;19:15-22.

51. Masalov VM, Sukhinina NS, Kudrenko EA, Emelchenko GA. Mechanism of formation and nanostructure of Stöber silica particles. Nanotechnology. 2011;22:275718-26.

52. Tsigkou O, Labbaf S, Stevens MM, Porter AE, Jones JR. Monodispersed bioactive glass submicron particles and their effect on bone marrow and adipose tissue-derived stem cells. Adv Healthc Mater. 2014;3:115-25.

53. Bari A, Bloise N, Fiorilli S, Novajra G, Vallet-Regí M, Bruni G, Torres-Pardo A, González-Calbet JM, Visai L, Vitale-Brovarone C. Copper-containing mesoporous bioactive glass nanoparticles as multifunctional agent for bone regeneration. Acta Biomater. 2017;55:493-504.

54. Stöber W, Fink A, Bohn E. Controlled growth of monodisperse silica spheres in the micron size range. J Colloid Interface Sci. 1968;26:62-9.

55. Bantz C, Koshkina O, Lang T, Galla HJ, Kirkpatrick CJ, Stauber RH, Maskos M. Beilstein. The surface properties of nanoparticles determine the agglomeration state and the size of the particles under physiological conditions. J Nanotechnol. 2014;5:1774-86.

56. Wu C, Fan W, Chang J. Functional mesoporous bioactive glass nanospheres: synthesis, high loading efficiency, controllable delivery of doxorubicin and inhibitory effect on bone cancer cells. J Mater Chem B. 2013;1:2710.

57. Courrol LC, de Oliveira Silva FR, Gomes L. A simple method to synthesize silver nanoparticles by photo-reduction. Colloids Surf A Physicochem Eng Asp. 2007;305:54-7.

58. Schumacher M, Reither L, Thomas J, Kampschulte M, Gbureck U, Lode A, Gelinsky M. Calcium phosphate bone cement/mesoporous bioactive glass composites for controlled growth factor delivery. Biomater Sci. 2017;5:578-88.

59. Balamurugan A, Sockalingum G, Michel J, Fauré J, Banchet V, Wortham L, Bouthors S, Laurent-Maquin D, Balossier G. Synthesis and characterisation of sol gel derived bioactive glass for biomedical applications. Mater Lett. 2006;60:3752-7.

60. Hoppe A, Meszaros R, Stähli C, Romeis S, Schmidt J, Peukert W, Marelli B, Nazhat SN, Wondraczek L, Lao J, Jallot E, Boccaccini AR. In vitro reactivity of $\mathrm{Cu}$ doped $45 \mathrm{~S} 5$ Bioglass $^{\circledR}$ derived scaffolds for bone tissue engineering. J Mater Chem B. 2013;1:5659-74.

61. Bejarano J, Caviedes P, Palza H. Sol-gel synthesis and in vitro bioactivity of copper and zinc-doped silicate bioactive glasses and glass-ceramics. Biomed Mater. 2015;10:25001-13.

62. de Oliveira AAR, de Carvalho BB, Mansur HS, de Magalhães M. Pereira. Synthesis and characterization of bioactive glass particles using an ultrasound-assisted sol-gel process: Engineering the morphology and size of sonogels via a poly (ethylene glycol) dispersing agent. Mater Lett. 2014;133:44-8.

63. Pishbin F, Mourino V, Flor S, Kreppel S, Salih V, Ryan MP, Boccaccini AR. Electrophoretic deposition of gentamicin-loaded bioactive glass/chitosan composite coatings for orthopaedic implants. ACS Appl Mater Interfaces. 2014;6:8796-806.

64. Atiq M, Rehman U, Bastan FE, Nawaz Q, Boccaccini AR. Electrophoretic deposition of lawsone loaded nanoscale silicate glass / chitosan composite on PEEK / BG layers. Electrochem Soc Tras. 2018;82:45-50.

65. Zheng K, Boccaccini AR, Sol-gel processing of bioactive glass nanoparticles. Adv Colloid Interface Sci. 2017;249:363-73. https://doi.org/10.1016/j.cis.2017.03.008.

66. Kaya S, Cresswell M, Boccaccini AR. Mesoporous silica-based bioactive glasses for antibiotic-free antibacterial applications. Mater Sci Eng C. 2018;83:99-107.

67. Ciapetti G, Savarino L, Miola M, Verné E, Vitale-Brovaron C, Baldini N. In vitro testing of manganese-doped bioglasses to stimulate osteoblast behaviour. Dental Materials. 2014;30:e166 